\title{
Transitional B cells commit to marginal zone B cell fate by Taok3-mediated surface expression of ADAM10
}

Hamida Hammad ${ }^{1,2,9}$, Matthias Vanderkerken ${ }^{1,2,9}$, Philippe Pouliot ${ }^{1,2,9}$, Kim Deswarte ${ }^{1,2}$, Wendy Toussaint ${ }^{1,2}$, Karl Vergote ${ }^{1,2}$, Lana Vandersarren ${ }^{1,2}$, Sophie Janssens ${ }^{1,2}$, Ioanna Ramou $^{1,3}$, Savvas N. Savvides ${ }^{1,3}$, Jody J. Haigh ${ }^{1}$, Rudi Hendriks ${ }^{4}$, Manfred Kopf ${ }^{5}$, Katleen Craessaerts $^{6}$, Bart de Strooper ${ }^{6}$, John F. Kearney ${ }^{7}$, Daniel H. Conrad ${ }^{8}$ \& Bart N. Lambrecht ${ }^{1,2,4}$

${ }^{1}$ VIB Inflammation Research Center, B-9000 Gent, Belgium

2Department of Respiratory Medicine, University Gent, B-9000 Gent, Belgium

${ }^{3}$ The Laboratory for Protein Biochemistry and Biomolecular Engineering (L-Probe), University Gent, B-9000 Gent, Belgium

${ }^{4}$ Department of Pulmonary Medicine, ErasmusMC, Rotterdam, The Netherlands ${ }^{5}$ Institute for Molecular Health Sciences, ETH, 8093 Zürich, Switzerland

${ }^{6}$ VIB Center for the Biology of Disease, VIB, B-2000 Leuven, Belgium

${ }^{7}$ Department of Microbiology, University of Alabama at Birmingham, Birmingham, AL 35294, USA

${ }^{8}$ Center for Clinical and Translational Research, Virginia Commonwealth University, Richmond, VA 23298, USA

${ }^{9}$ These authors contributed equally

Correspondence should be addressed to B.N.L. (bart.lambrecht@irc.vib-Ugent.be)

Bart N. Lambrecht, MD, PhD

VIB Inflammation Research Center, Ghent University

Technology Park 927

9000 Gent, Belgium

+3293313607 
Notch2 and $B$ cell antigen receptor (BCR) signaling determine if transitional $B$ cells become marginal zone $B$ (MZB) or follicular B (FoB) cells in the spleen, but it is unknown how these pathways are related. We generated Taok3-1- mice and found cell-intrinsic defects in the development of MZB, but not FoB cells. Type 1 transitional (T1) B cells required Taok3 to rapidly respond to ligation with the Notch ligand Delta-like 1. BCR ligation by endogenous or exogenous ligands induced the surface expression of the metalloproteinase ADAM10 on T1 cells in a Taok3dependent manner. T1 B cells expressing surface ADAM10 were committed to become MZB cells in vivo, whereas T1 B cells lacking expression of ADAM10 were not. Thus, during positive selection in the spleen, BCR signaling causes immature T1 cells to become receptive to Notch ligands via Taok3-mediated surface expression of ADAM10. 


\section{Introduction}

B lymphocytes are categorically divided in B1 and B2 cells. B1 cells derive from fetal progenitors and react to a restricted set of microbial ligands in a T cell-independent ( $\mathrm{TI})$ manner in serosal cavities and spleen ${ }^{1}$. B2 cells develop continuously in the bone marrow and further mature into follicular B (FoB) cells and marginal zone B (MZB) cells. FoB cells have a broad repertoire of specificity, recirculate between lymphoid organs and give rise to germinal center $B$ cells that undergo somatic hypermutation in a T-cell dependent (TD) manner. MZB cells shuttle continuously between the marginal zone and follicles of the spleen and produce antibodies to encapsulated bacterial and polysaccharide $\mathrm{TI}$ antigens. It is still poorly understood how immature transitional $B$ cells are instructed to become a FoB or MZB cell, and when exactly this lineage choice is made. It is widely accepted that MZB instruction requires triggering of Notch2 on developing B cells by Delta-like 1 (DIl1) expressed by splenic red pulp sinus endothelial cells or marginal zone reticular cells (MRCs). Notch2 cleavage by a metalloproteinase and disintegrin-10 (ADAM10) and $\gamma$ secretase then releases the intracellular domain of Notch (NICD) that binds to the transcription factor RBP-JK in the nucleus, and instructs MZB development, together with $\mathrm{NF}-\mathrm{kB}$ signaling emanating from the BAFF receptor ${ }^{2-13}$. The quality of $\mathrm{B}$ cell antigen receptor $(B C R)$ signals during positive selection of $B$ cell precursors in the spleen is equally important in $B$ cell fate decisions ${ }^{14-16}$, and it was proposed that weak or strong BCR signals might render cells receptive or resistant to Notch instruction ${ }^{17,}{ }^{18}$. Yet how BCR repertoire or signaling controls Notch responsiveness is currently poorly understood. The Ste20 family kinases are serine-threonine kinases that participate in a variety of signaling pathways triggered by cellular stress ${ }^{19}, 20$. The Tao kinase subfamily has three members in mammals (TAOK1, also known as proteins MAP3K16, PSK2 or MARKK; TAOK2 (MAP3K17, PSK1) and TAOK3 (MAP3K18, JIK or DPK)), whose function is

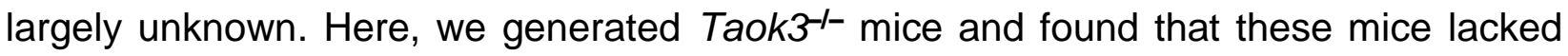
MZB cells, whereas FoB cells were intact. By carefully unraveling the molecular mechanism of this deficiency we have discovered how BCR signaling intersects with Notch signaling in immature transitional B cells undergoing positive selection.

\section{RESULTS}




\section{Taok3-- mice lack MZB cells}

In wild-type mice, the expression of mRNA for Taok3 was predominantly found in bone marrow and immune tissues like spleen, thymus and lymph nodes, but also in lung and gut (Fig. 1a). To gain insight into the biology of Taok3, we generated Taok3 $3^{-1-}$ mice (Supplementary Fig. 1a-e). Overall there was no difference in the cellularity of the various lymphoid organs in 6-12 week old mice (Supplementary Fig. 1f). In the spleen, there were no gross differences in the percentage of eosinophils, monocytes, natural

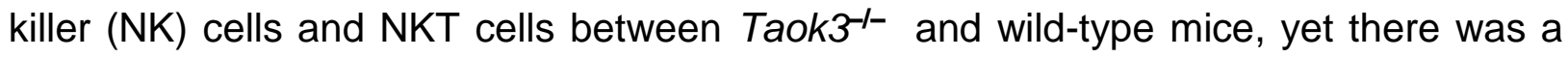

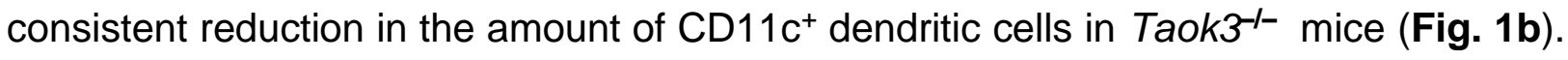
There was a small yet consistent increase in the percentage of $\mathrm{Ly}_{6 \mathrm{G}} \mathrm{G}^{+}$granulocytes in the spleen of Taok3 $^{-/}$mice. The distribution of $\mathrm{CD}^{+} \mathrm{T}$ cells and $\mathrm{CD} 19^{+} \mathrm{B}$ cells was comparable, yet there was a $25-30 \%$ reduction in the amount of CD8 ${ }^{+} \mathrm{T}$ cells (Fig. 1c). Within the B cells of the spleen, analysis of cell surface expression of CD21/35 and CD23 discriminates between transitional $C D 21 / 35^{-} C D 23^{-} B$ cells that are immature $B$ cells that have just arrived from the bone marrow, CD21/35 $5^{\text {int }} C D 23^{\text {hi }}$ FoB cells and CD21/35 hiCD23- MZB cells. Transitional B cells express CD93 and can be further divided

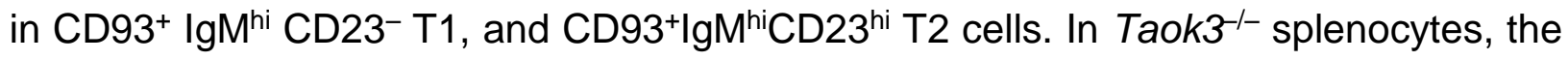
percentage of $\mathrm{T} 1, \mathrm{~T} 2$, and FoB cells were comparable to wild-type, whereas MZB cells were almost completely absent (Fig. 1d-f).

Histological examination of the spleen revealed that the characteristic rim of $\operatorname{lgM}^{+} \mathrm{CD} 1 \mathrm{~d}^{+}$ MZB cells separated from the $\lg ^{10} B$ cell follicles by the marginal sinus was absent in Taok3- mice (Fig. 1g). Resident marginal zone metallophillic macrophages (MMM, expressing CD169) that line the marginal sinus were present and correctly localized in

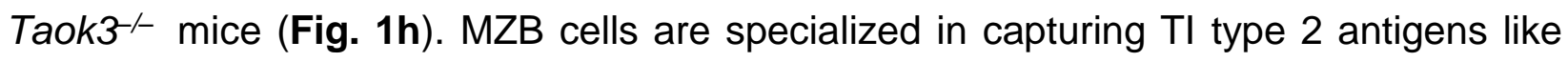
Ficoll ${ }^{21}$. Two $h$ after i.v. injection of FITC-Ficoll, we detected labeled B cells in vicinity of

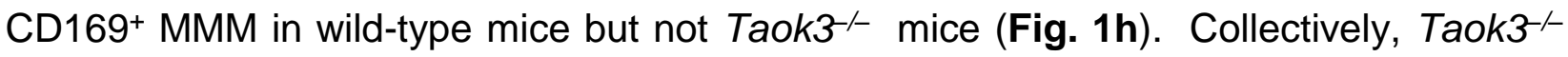
mice lack MZB cells without compensatory alterations in other $B$ cell subsets.

\section{Humoral immune response of Taok3 ${ }^{--}$mice}

The baseline serum concentration of immunoglobulins (lg) was comparable to wild-type, with a tendency for increased IgG3 in Taok3-- mice (Supplementary Fig 2). MZB cells 
acutely produce $\mathrm{TI}$ Ig to polysaccharide particulate antigens like Ficoll ${ }^{1,22}$. However, in response to immunization with trinitrophenyl (TNP) hapten-conjugated Ficoll, there was no reduction in the concentration of TNP-specific IgG1, IgG3 and IgM in Taok3-compared with wild-type mice (Fig. 1i and Supplementary Fig 2 ). The intact TNPspecific Ig response as not due to compensatory increase in recirculating MZB cells outside the spleen (data not shown). B1 B cells can also respond to TNP-Ficoll antigen, in the complete absence of MZB cells ${ }^{2}$. The numbers of B220 ${ }^{+} \mathrm{CD} 5^{+} \mathrm{B} 1 \mathrm{a}$ and CD5- B1b $B$ cells of the peritoneal cavity were identical in wild-type and Taok3 ${ }^{-}$mice (Fig. 1j), which may explain the intact humoral immune response to TNP-Ficoll. MZB cells are indispensable for mounting low affinity IgM phosphorylcholine (PC)-specific antibody responses to encapsulated bacteria like Streptococcus pneumonia when these reach the bloodstream ${ }^{1,2,23}$. We injected $1 \times 10^{8}$ heat inactivated pneumococci i.v. and measured the IgM response antigen 5 days later. Whereas wild-type mice readily mounted an antiPC IgM response, the titer of PC-specific IgM was severely reduced in Taok3 ${ }^{-/}$mice (Fig.

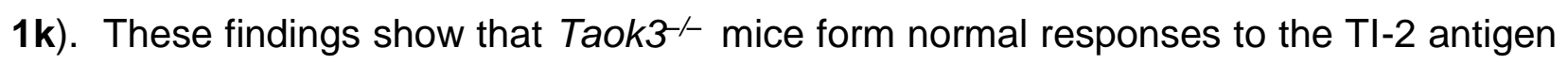
Ficoll, but not to the TI-1 antigen S. pneumoniae.

\section{MZB defect of Taok3- mice is B cell-intrinsic}

The development and survival of MZB cells depends on integrin signaling and correct positioning in the marginal zone ${ }^{18,24-26}$. As the structure of the marginal zone was not normal in Taok3 ${ }^{-1}$ mice, and other defects were found in neutrophils, DCs and CD8 ${ }^{+} \mathrm{T}$ cells, we next addressed if the defect in MZB cell development was cell intrinsic, or caused by changes in the stromal structures or other hematopoietic cells. We therefore created mixed bone marrow chimeric mice by lethally irradiating CD45.1.CD45.2 C57BL/6 mice and reconstituting them with an equal mix of CD45.1 wild-type and CD45.2 Taok3 ${ }^{-}$BM cells. Chimerism was complete 6-8 weeks after transfer with each donor genotype contributing to $50 \%$ of monocytes and neutrophils in the blood and various organs (data not shown). Whereas CD23 ${ }^{\text {hi }} \mathrm{CD} 21 / 35^{\text {lo }}$ Fo B were completely chimeric (Fig. 2a), $\mathrm{CD} 21^{\text {hi }} \mathrm{CD} 23^{\text {lo }} \mathrm{MZB}$ cells were generated almost exclusively from the CD45.1 wild-type hematopoietic cells. As the marginal zone was fully restored in chimeric mice (data not shown), these findings demonstrate that the defect in MZB development is not due to 
changes in environment, but cell-intrinsic. We next evaluated at which stage of B cell development the MZB development might be compromised. In the bone marrow of chimeric mice, pro-B cells, cycling pre-B cells, pre-B cells and immature $B$ cells were all equally distributed amongst both genotypes (Fig. 2b). In the spleen however, there was an overrepresentation of T1 cells of the CD45.1 wild-type genotype. This effect was less marked in T2 B cells. Collectively, loss of MZB cells in Taok3-- mice is cell intrinsic and $\mathrm{B}$ cell development is compromised from the T1 stage onwards.

\section{Gene dosage effect of Taok3 deficiency}

To address if the amount of Taok3 affects B cell development, we analyzed heterozygous Taok3 ${ }^{+-}$mice and took advantage of the fact that the gene trap construct made to inactivate the Taok3 locus contained a splice acceptor that was flanked by loxP sites, allowing the partial reversal of the gene trap in cells of interest (Supplementary Fig. 1a).

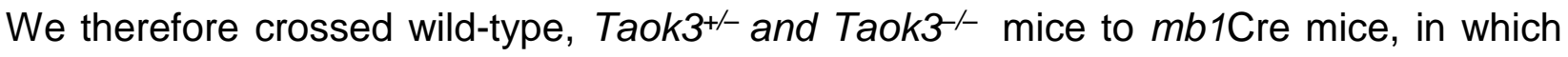
Cre recombinase expression is under the control of the Cd79a promoter, active from the pre $B$ cell stage onwards ${ }^{27}$. When we analyzed the composition of splenic $B$ cells in wildtype $\mathrm{Cre}^{+}, \mathrm{Taok}^{-/} \mathrm{Cre}^{-}$or $\mathrm{Taok}^{-/} \mathrm{Cre}^{+}$mice, we found that MZB cells were partially recovered by gene-trap reversal exclusively in the B cell lineage (Fig. $\mathbf{2 c}, \mathbf{d}$ ). The numbers of MZB cells of heterozygous Taok3 ${ }^{+/}$were intermediate between those of wild-type and

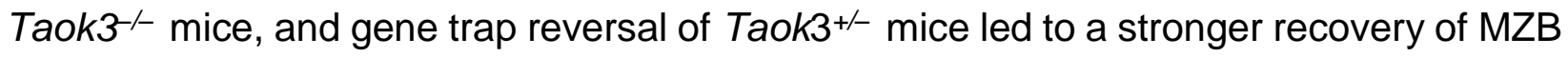
cells compared with reversal in Taok3 ${ }^{-}$mice. Collectively, the defect in MZB development in Taok3 ${ }^{-}$mice can be partially reversed by B cell specific gene trap removal.

\section{Defective Notch activation in Taok3 ${ }^{-/}$B cells}

The gene dose-dependent lack of MZB cells and preserved induction of immune responses to TNP-Ficoll in Taok3 ${ }^{-}-$mice resembles the phenotype of mice lacking Notch2 or RBP-JK in the B cell lineage ${ }^{2,6,7}$. It has been shown that transitional $B$ cells are instructed to become MZB cells via interaction with the Notch ligand Delta-like 1 (DII1) expressed on stromal cells ${ }^{4,5}$. To address if Notch-mediated development of MZB cells was disrupted in Taok3 $^{-/}$mice, we cultured $\mathrm{CD}^{-} 3^{+}$transitional splenic B cells on OP9- 
GFP cells, or on OP9 cells stably transfected with Delta-like 1 (OP9-DIl1). Notch signaling was studied by measuring the mRNA of direct Notch targets genes Dtx1, Hes1, Hes5 and Hey ${ }^{11}$. When transitional B cells were cultured for 4h on OP9-Dll1 cells, there was induction of Notch target genes in wild-type but much less efficiently so in Taok3-- mice (Fig. 3a). Strikingly, these differences were no longer apparent 18h later (data not shown), demonstrating that Taok3 was mainly involved in controlling rapid responsiveness to Dll1 ligation.

It is exceedingly difficult to model MZB development in vitro ${ }^{28,29}$. However, when wildtype $\mathrm{CD}^{+} 3^{+}$transitional B cells were co-cultured with OP9-GFP cells in the presence of the B cell growth factor BAFF, there was induction of CD21 and IgM on $8-10 \%$ of $T a o k 3^{+/+}$ cells. Culture on OP9-DIl1 induced expression of IgM and CD21 on roughly $20 \%$ of cells. However, Taok3 ${ }^{-}$transitional B cells only upregulated CD21 and IgM on $10 \%$ of B cells when cultured on OP9-Dll1 cells (Fig. 3b). These differences were not due to alterations in BAFF signaling (Supplementary Fig. 3a) or BAFF induced survival (Supplementary Fig. 3b). Thus, Taok3 was necessary in transitional $B$ cells for rapid Notch signaling and differentiatiation towards the MZB phenotype.

To address this point more directly, we performed a rescue experiment in which Notch2

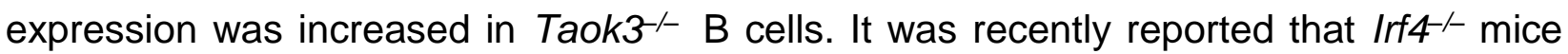
have an increase in MZB cells explained through a stabilization of intracellular Notch2 ${ }^{12}$. We set up breedings of $\mathrm{Taok3}^{-/}$and $I \mathrm{rft}^{-/}$mice. We confirmed that $I \mathrm{rft}^{-/}$mice had a

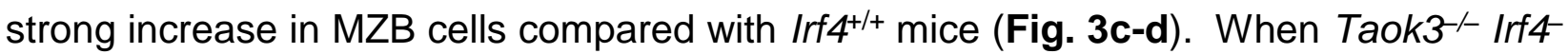
- mice were analyzed, the deficiency of MZB cells was completely reversed, and there were even higher percentages MZB cells compared with wild-type animals. We validated that Taok3 ${ }^{-}$mice did not have an increased intracellular accumulation of IRF4 protein (data not shown). These data show that Taok3-deficiency leads to defective Notch

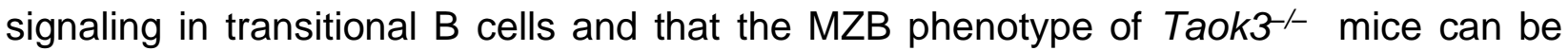
rescued by IRF4 deficiency.

\section{Taok3 controls the surface expression of ADAM10}


We next studied how lack of a kinase might lead to reduced Notch signaling. By immunoblot analysis on lysates, Notch2 expression in Taok3-- was similar to wild-type mice (Supplementary Fig 4). Looking at immunostained splenic sections and flow cytometry we found that the intensity of CD23 staining on B cell follicles (Fig. 4a) and FoB

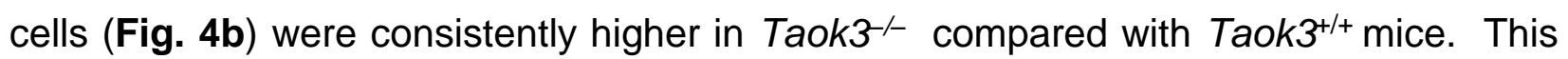
was not caused by altered Cd23 mRNA (data not shown). We have previously shown that the metalloproteinase ADAM10 determines the intensity of CD23 on the B cell plasma membrane, by cleaving CD23 to generate a sCD23 fragment ${ }^{10,30}$. We found that the

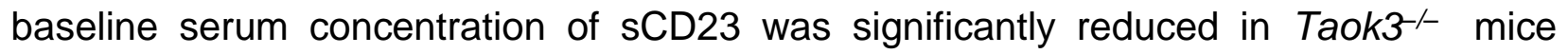
compared with wild-type mice (Fig. 4c). Injection of the 19G5 antibody against the CD23 stalk causes a conformational change in CD23 rendering it highly sensitive to cleavage by ADAM1010. After $19 \mathrm{G} 5$ injection in wild-type mice, there was an almost 100 -fold increase in the serum levels of SCD23. This effect was strongly reduced in Taok3- ${ }^{-}$mice, suggesting a defect in the enzymatic activity of ADAM10 (Fig. 4d). The defect in ADAM10 bioactivity was addressed further by studying the cleavage of other ADAM10 substrates. A well-known substrate of ADAM10 is the amyloid precursor protein (APP), involved in the pathogenesis of Alzheimer's disease ${ }^{31}$. The ADAM10 causes the cleavage of cell bound APP into a membrane bound APP $\alpha$-stub and the release of soluble APP $\alpha$. As APP is produced by mouse fibroblasts as well as neuronal cells, we generated mouse embryonic fibroblast (MEF) cell lines of wild-type and Taok3 ${ }^{-/}$mice and found that Taok3/ MEFs had a consistently lower expression of the APP $\alpha$-stub on their cell surface, suggestive of reduced ADAM10 activity in Taok $3^{-}$mice. When MEFs were transfected with full length APP, we could also measure the release of SAPP $\alpha$ in the concentrated supernatant, and found that MEFs derived from Taok3- ${ }^{-}$mice generated less SAPP $\alpha$ compared with $\mathrm{Taok}^{+/+}$mice, suggestive indeed of reduced ADAM10 bioactivity (Supplementary Fig. 5).

ADAM10 is known to cleave substrates when expressed on the cell surface, and we have reported that ADAM10 can be detected by flow cytometry on lymphocytes ${ }^{10}$. We found that $5-6 \%$ of $C D 93^{+}$transitional $B$ cells of the spleen of wild-type mice expressed ADAM10 on their cell surface, yet this expression was almost completely absent in $\mathrm{Taok}^{-/}$mice 
(Fig. 4e). However, the expression of the pool of ADAM10, composed of pro- and of mature ADAM10, in cell lysates of transitional $B$ cells was identical between wild-type and

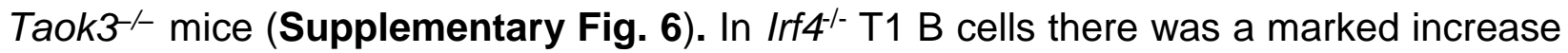

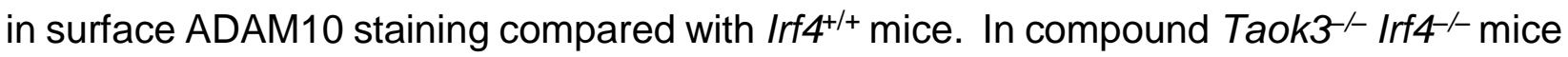
the defect of ADAM10 expression seen in Taok3 ${ }^{-}$mice was restored well above the intensity seen in Taok3 wild-type mice, suggesting that lack of IRF4 restored MZB numbers through upregulation of ADAM10 on T1 B cells (Fig. 4f). There was a strong correlation between the number of T1 cells expressing surface ADAM10 and the final pool of mature MZB cells in mice of various genotype (Fig $\mathbf{4 g}$ ). Collectively, Taok3 ${ }^{-/-}$mice lacked surface ADAM10 on T1 B cells and the return of ADAM10 on T1 B cells was accompanied by return of MZB cells, suggesting that the expression of ADAM10 on the surface of might be a crucial event in MZB development

\section{ADAM10 marks MZB commitment in T1B cells}

The lineage choice of transitional B cells to become FoB or MZB cell depends on the repertoire of the $B C R$ and the strength of $B C R$ signaling ${ }^{14-16}$. We therefore reasoned that positive selection events of MZB cells acting through the BCR in transitional $B$ cells might affect the surface expression of ADAM10. To test this, we first purified $\mathrm{CD} 93^{+}$transitional $B$ cells and stimulated them with soluble BCR crosslinking using anti-lgM $F\left(a b^{\prime}\right) 2$ fragments. In unstimulated wild-type cells, ADAM10 expression was mainly found in discrete punctate area inside transitional B cells. Within 20 min following BCR stimulation, there was a relocalization of ADAM10 to a single cap-like region on the $B$ cell, a lipid-raft and tetraspanin rich region concentrating the $\mathrm{BCR}$, $\lg \alpha$, Ras, and $\mathrm{BLNK}^{32}$. Simultaneous staining for IgM revealed colocalization with ADAM10 with the capped BCR (data not

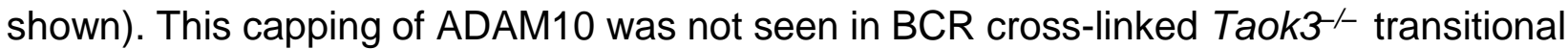
B cells (Fig. 5a).

We next studied if positive selection in a more physiological context would also affect ADAM10 surface expression on T1 B cells. Previously, we have created transgenic mice expressing the VH81x heavy chain, that pairs with a limited repertoire of endogenous light 
chains through binding constraints, thus generating a BCR that recognizes an endogenous self- or microbiome-derived ligand. These mice have been used to understand the mechanisms of positive selection of cells in the MZB pool ${ }^{14}$. We crossed Taok3 $3^{-}$mice to $\mathrm{VH} 81 \times \mathrm{Tg}$ mice, and stained spleen sections for the presence of clonotype specific B cells (Fig 5b). In VH81x Tg mice with wild-type levels of Taok3, the clonotype ${ }^{+}$cells were almost exclusively found in the splenic marginal zone, whereas in VH81x Tg Taok3 ${ }^{--}$mice, these cells were absent. Flow cytometry also showed massive expansion of MZB cells in VH81x Tg mice, yet when Taok3 was inactivated, MZB cells were absent (Fig. 5c). We also performed flow cytometry using antibodies recognizing the $\mathrm{VH} 81 \mathrm{x}$ Ig heavy chain and the pairing $\mathrm{V}_{\kappa} 1 \mathrm{C}$ light chain. This staining revealed that in Taok $3^{+/+} \mathrm{VH} 81 \times \mathrm{Tg}$ mice the majority of the clone was found in the CD21 ${ }^{\text {hi }}$ MZB cell gate,

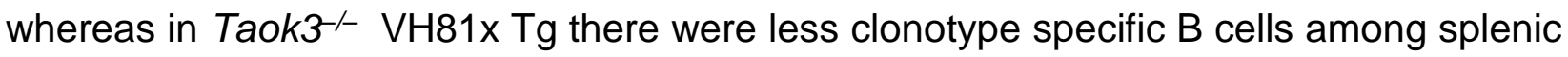
CD19+ cells, and the majority of remaining clonotype specific cells had a FoB cell phenotype (Fig $\mathbf{5 d - e}$ ). The percentage of T1 cells expressing surface ADAM10 was strongly increased in VH81x Tg mice, an effect that was abolished by crossing these mice to Taok3 ${ }^{-}$mice (Fig. 5f). Again there was a strong correlation between the percentage of mature MZB cells and the percentage of surface ADAM10+ 11 cells in mice across all genotypes (data not shown).

We finally reasoned that expression of ADAM10 on T1B cells might mark commitment of these cells to become MZB cells. The high numbers of T1 B cells in the spleens of VH81x $\mathrm{Tg}$ mice allowed us to obtain sufficient cells to perform adoptive transfer experiments into B cell-deficient Rag2/- mice. We therefore purified CD45.2+ ${ }^{+}$D $93^{+}$transitional $\mathrm{B}$ cells by magnetic pre-enrichment and subsequently sorted cells into ADAM10 surface-positive CD23-T1B cells, ADAM10 surface-negative CD23- T1B cells, and CD23+ T2B cells (which do not express high levels of ADAM10 anyway, see fig. 4e). These were injected simultaneously with ten times higher numbers of CD45.1 wild type splenic cells, to avoid homeostatic proliferation that might bias to MZB development (Fig. $\mathbf{5 g}$ ). Five days after transfer, the fate of transferred CD45.2 cells was studied by flow cytometry on spleen cells and confocal analysis of the spleen. Whereas T2B cells and ADAM10 surface-negative T1 $B$ cells gave rise to both FoB and MZB cells, the ADAM10 surface-positive T1 B cells exclusively gave rise to CD21 ${ }^{\text {hi }}$ CD23- MZB cells (Fig. 5h). Histological analysis of the 
spleen also revealed that these CD45.2+ $A D A M 10^{+} \mathrm{T} 1$ derived MZB cells were predominantly found in the marginal zone of the spleen (Fig. 5i). Previously, others have proposed that preMZB cells can be identified amongst a pool of TB cells, and that these cells exhibited higher expression of CD21 and CD1d, but it is unclear if this would be a $\mathrm{T} 1 \mathrm{~B}$ or $\mathrm{T} 2 \mathrm{~B}$ stage $^{6,16}$. We found that $\mathrm{T} 1 \mathrm{~B}$ cells that expressed surface levels of ADAM10 were higher in the expression of CD21 and CD1d, compared with T1B cells lacking ADAM10 surface expression (Supplementary Fig 7). These data confirm our hypothesis that ADAM10 surface expression marks progenitor T1B cells committed to become MZB cells after positive selection. 


\section{Discussion}

Our understanding of the development of splenic MZB from immature transitional B cells is dominated by three lines of thought ${ }^{18}$. First, integrin and chemokine signals are crucial for retention and complex shuttling of MZB cells in and around the marginal zone. Loss of these interactions led to loss of MZB cells in many mouse strains ${ }^{21,25,26,33}$. Secondly, Notch2 ligation by DII1 expressed on stromal cells is required for MZB development ${ }^{2-13}$, and maintenance of MZB identity ${ }^{12,34}$. Thirdly, the quality and the strength of the BCR repertoire determine whether positive selection of immature $B$ cells by self ligands or microbiome derived ligands leads to deletion, FoB cell or MZB development ${ }^{14-16,35}$. It has been unclear how these three pathways are related.

Pillai et al. proposed that strong BCR signals favour FoB, whereas weak BCR signals promote MZB cell development ${ }^{17,18,36,37}$, although other investigators refuted this idea ${ }^{11}$, 15. It was proposed that strong BCR signals render transitional cells in the follicle impervious to the presence of Dll1 mediated triggering of Notch2, whereas weak BCR signaling may enhance the expression of one or more components of the Notch2 signaling pathway ${ }^{36}$. These inhibitory or enhancing signals or the precise stage of $\mathrm{B}$ cell development where BCR signaling and Notch permissiveness intersect have never been identified to date. Here, by careful analysis of Taok3 ${ }^{-1}$ mice we show that BCR mediated positive selection of $B$ cell progenitors at the T1B cell stage is linked to MZB development through acquisition of membrane expression of ADAM10 that cleaves and activates Notch2. ADAM10 is expressed on a subset of transitional B cells, is redistributed to the immunocap following BCR crosslinking, and highly expressed on the surface of T1B cells. We believe that the $5-10 \%$ of ADAM10+ transitional $B$ cells that are found in the steady state represent the cells that are undergoing positive selection to become MZB cells, supported by our observation that these cells only become MZB cell upon adoptive transfer.

How exactly B cell positive selection and BCR signaling intermediates cause Taok3 activation and ADAM10 surface expression will require further study. The levels of Taok3mRNA remain stable throughout B cell development from hematopoietic cells, and are not altered during $B$ cell activation (data not shown) suggesting that Taok3 might be 
mainly regulated by posttranslational modifications or protein stabilization. It was recently proposed that ADAM10 forms a homodimer in the cell membrane as does ADAM17, a key feature in the proposed regulation mechanism of ADAM activation ${ }^{38}$. Although we did observe differences in the processing and oligomerization of ADAM10 in Taok3- mice (data not shown), more research is warranted. Much more is known about the regulation of ADAM17, which can also cleave Notch. The phosphorylation of ADAM17 by ERK kinase on Threonine735 regulates ADAM17 dimerization and enzymatic activity ${ }^{39}$. A conserved threonine in position 719 might have the same effect on dimerization of ADAM10, a hypothesis we are currently testing. Taok3 is a serine/threonine MAP3 Kinase and is likely upstream of ERK, JNK or p38 Map Kinase (unpublished observations and ${ }^{40}$ ). Although it is likely therefore that lack of MAPK mediated ADAM10 surface expression is the explanation for the MZB phenotype of Taok3-deficient mice, we also need to consider additional effects of Taok3 on MAPK-driven activation of NF-kB, as canonical NF-kB1 collaborates with Notch 2 in driving MZB fate determination ${ }^{8}$.

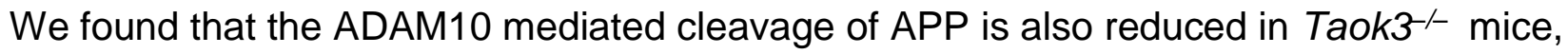
suggesting that the $B C R$ is not the only upstream regulator of Taok3 and ADAM10 bioactivity. However, Taok3 is not absolutely or always required for ADAM10 or Notch2 activation, as Taok3 $^{-/}$mice do not phenocopy all aspects of ADAM10 or Notch2

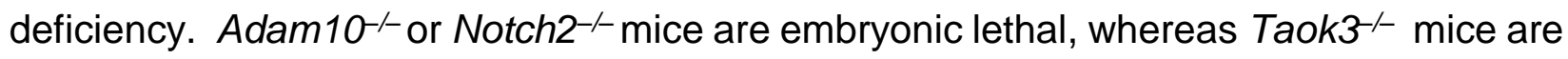
not. Our in vitro findings using OP9-DIl1 cells to stimulate transitional B cells suggest that the phenotype of Taok3 ${ }^{-}$mice is the result of disruption of short-lived Notch-Notch-ligand interactions. Such short-lived interactions might occur on the MRC network of the B cell follicles, and might be restricted in time due to the continuous shuttling behavior of MZB cells from the $M Z$ to the $B$ cell follicles ${ }^{5,24}$.

Our data resolve a longstanding confusion in the field as to the precise identity of MZB precursors. It was long held that MZB cells emanate from progenitors with a CD23+ $T 2 B$ phenotype that express $C D 1 d$ and $C D 21^{6,10,17,41}$. Others have also proposed that FoB cells can develop into MZB cells, particularly in immunodeficient and lymphopenic hosts, a process that might be driven by homeostatic proliferation ${ }^{24,41,42}$. Data from mice in which the repertoire of developing MZB cells was followed by measuring the junctional diversity 
of the heavy chain CDR3 region revealed that T1B cells were the most likely direct progenitors for MZB cells ${ }^{16}$. Our data using adoptive transfer, as well as the strong correlations between ADAM10 expression on T1B cells and final population size of the MZB pool suggest that indeed commitment of MZB cells is made at the T1B cell stage, and that surface ADAM10 staining is a robust marker for identifying cells in which MZB commitment is complete. However, we could show that some T2B cells could still differentiate into MZB cells. We predict that also those T2B cells upregulate ADAM10 surface expression after adoptive transfer in vivo ${ }^{10}$.

In conclusion, we have shown that signals from the BCR control MZB lineage choice by regulating the surface expression of the metalloproteinase ADAM10 that has the potential to cleave and activate Notch2 in T1 transitional B cells undergoing positive selection. 


\section{ACKNOWLEDGEMENTS}

H.H. is supported by Ghent University Grant (GOA 01G02817). M.V. is supported by a FWO Fellowship (grant 3F023515W). P.P was supported by Marie Curie grant (MC 237581). S.J. is supported by FWO grant G085915N and a University of Ghent (MRPGROUP-ID). M.K. is supported by Swiss National Science Foundation (SNF 310030163443/1). J.F.K. was supported by NIAID Al14782 and Al100005. B.N.L. was supported by an ERC consolidator grant 261231 and a University of Ghent Multidisciplinary Research Platform (MRP-GROUP-ID) and Ghent University Grant (GOA 01G02817).

\section{AUTHOR CONTRIBUTIONS}

Hamida Hammad designed, performed and analyzed experiments and wrote the manuscript

Matthias Vanderkerken performed and analyzed experiments and wrote the manuscript

Philippe Pouliot performed and analyzed experiments

Kim Deswarte performed and analyzed experiments

Wendy Toussaint performed and analyzed experiments

Karl Vergote performed and analyzed experiments

Lana Vandersarren performed and analyzed experiments

Sophie Janssens performed and analyzed experiments

Ioanna Ramou performed and analyzed experiments

Savvas N. Savvides performed and analyzed experiments

Jody J. Haigh constructed mice

Rudi Hendriks performed and analyzed experiments

Manfred Kopf provided crucial tools and analyzed experiments

Katleen Craessaerts performed and analyzed experiments

Bart de Strooper provided crucial tools and analyzed experiments

John F. Kearney provided crucial tools and analyzed experiments

Daniel H. Conrad provided crucial tools and analyzed experiments

Bart N. Lambrecht conceived the study, designed and analyzed experiments and wrote the manuscript

COMPETING FINANCIAL INTERESTS

none 


\section{METHODS \\ Mice}

We generated Taok3- - mice from ES cells (clone CC0463 from the Sanger Institute Gene Trap Resource (SIGTR), Cambridge, UK) in which a loxP flanked splicing acceptor (SA) preceding a nuclear $\beta$-galactosidase-neomycin resistance cassette and poly $A$ tail was inserted as a gene trap in the intronic region between exon 1 and exon 2 of the Taok3 gene, leading to premature transcriptional termination. ES cells were originally made in Sv129 background, but germline transmitting mice were backcrossed for 10 generations

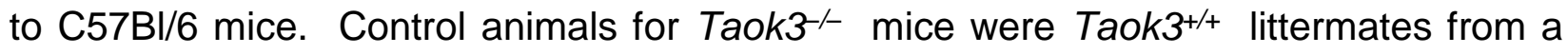
heterozygous Taok3 $3^{+-}$breeding. Irf4-/- mice were obtained from $\mathrm{Dr}$ W. Agace, Lund University, and were originally derived from The Jackson laboratory. VH81x mice expressing the VH81x heavy chain, that pairs with a limited repertoire of endogenous light chains through binding constraints have been previously described ${ }^{14}$. Mb1Cre mice, in which Cre recombinase expression is under the control of the Cd79a promoter, active from the pre B cell stage onwards were obtained from The Jackson laboratory and have been previously described ${ }^{27}$. Mice were maintained under specific pathogen free conditions. All animal experiments and procedures were approved by the local animal ethics committee of Ghent University.

\section{Flow cytometry}

Cells suspensions were obtained from the spleen, lymph nodes or the bone marrow of 68 week old mice through a $100 \mu \mathrm{m}$ mesh and red blood cell lysis. Surface stainings were performed in PBS using the following antibodies: CD19 (1D3; eBiosciences), CD93 (AA4.1; ebiosciences), CD23 (B3B4; eBiosciences), CD21/35 (4E3; eBiosciences), IgM (II/41; BD biosciences), CD1d (1B1; BD Biosciences), CD3 (17A2; eBiosciences), CD4 (RM4-5; ebiosciences), CD8 (53-6.7; eBiosciences), CD11c (N418; eBiosciences), MHCII (M5/114.15.2; eBiosciences), Ly-6G (1A8; eBiosciences), Siglec F (E50-2440; BD biosciences), NK1.1 (PK136; BD biosciences), ADAM10 (139712; R\&D systems).

For analysis of various B cell progenitors in the bone marrow, we stained red blood cell lysed bone marrow cells with antibodies to B220, CD43, CD24, CD19, lineage (CD3, Ly6G, CD11b, Ter119, NK1.1), according to a staining panel obtained from the Immgen 
consortium. For live-dead cell discrimination, we used a fixable viability dye eFluor506 (eBiosciences). Sample data were acquired with a 4 laser BD LSR Fortessa flow cytometer (BD Biosciences) using the BD FACSDiva Software. Data analysis was performed using FlowJo software (Treestar).

\section{Immunizations}

For experiments addressing humoral immune responses, mice ( $n=6$ per immunization and per genotype) were immunized with TNP-Ficoll (50 ug/ animal, i.v or i.p. in $200 \mathrm{ul} \mathrm{PBS}$ ) or TNP-KLH in alumunium hydroxide (100 $\mu \mathrm{g}$ / animal, i.p. + $1 \mathrm{mg}$ Alum in $200 \mu \mathrm{l}$ PBS) on day 0 , followed by a booster of TNP-KLH of $100 \mu \mathrm{g}$ at day 35. TNP-Ficoll and KLH-TNP were from Biosearch Technologies and alumunium hydroxide. Immune responses were read out at day 0 (baseline), 7 (for TNP-Ficoll), 35 (pre-booster) and 42 for TNP-KLH/alum by measuring serum immunoglobulin levels. TNP-specific lgG1, IgG2, IgG3 and IgM antibodies were measured by commercially available ELISA (Biosearch Technologies).

For measuring the response to Streptococcus pneumoniae, $1 \times 10^{8}$ heat inactivated pneumococci were injected intravenously into wild-type (WT) and Taok3/- mice and the IgM Ab response to the PC antigen 5 days later by commercially available ELISA.

Levels of SCD23 were measured in the serum at day 5 after 2 injections of $20 \mu \mathrm{g}$ of $19 \mathrm{G} 5$ antibody (directed against the stalk region of CD23) on day 0 and day2, by commercially available sCD23 ELISA (R\&D systems), as described ${ }^{10,30}$. This $19 \mathrm{G} 5$ antibody causes ADAM10-dependent cleavage of SCD23.

\section{Cleavage of amyloid precursor protein}

We generated mouse embryonic fibroblasts (MEF) from wild-type and Taok3 ${ }^{-/}$embryos.

\section{Stimulation of transitional B cells on OP9-DIl1 cells}

OP9-DIl1 or control OP9-GFP cells were grown to $80 \%$ confluence in 24 well plates in optimized medium as described in detail ${ }^{43}$. For enrichment of transitional B cells, red cell 
lysis was performed first on spleen suspensions, cells washed and stained using CD93APC. Cells were sorted using magnetic bead enrichment with anti-APC beads and LS columns (both from Miltenyi). Subsequently, $5 \times 10^{5}$ magnetically enriched CD93 (4AA.1)+ transitional B cells were added to the OP9 cells. RNA was extracted at 4 and $18 \mathrm{~h}$ after setting up the co-culture and cDNA was made using a commercially available kit (Transcriptor High Fidelty Kit, Roche). In some experiments, the B cell growth and survival factor BAFF ( $10 \mathrm{ng} / \mathrm{ml}$; R\&D systems) was added according to a published protocol ${ }^{29}$, and cells were harvested for flow cytometry 3 days later. Survival was analyzed using flow cytometry by adding DAPI to the cells.

\section{Confocal imaging}

Confocal imaging was performed on spleen sections or magnetically enriched transitional $B$ cells. The following antibodies were used: ADAM10 (clone EPR5622, rabbit polyclonal) was purchased from Abcam. IgM (II/41), B220 (RA3-6B2), CD45.2 (142) and CD1d (1B1) were obtained from BD Biosciences. CD3 (17A2) was obtained from ebiosciences. CD169 (MOMA-1) was obtained from Serotec Biorad. Briefly, $7 \mu \mathrm{m}$ spleen frozen sections or purified $\mathrm{CD}_{3} 3^{+}$transitional cells were fixed for 5 minutes in PFA 4\%. After washing with PBS, sections were stained with the primary antibodies for 60 minutes at room temperature, followed by a 30 minute-incubation period with secondary antibodies (all obtained from Jackson Immunoresearch). For visualizing the NP-reactive pool of MZB cells, we injected FITC-labeled Ficoll (Biosearch Technologies) intravenously. After two hours mice were euthanized and the distribution of FITC-Ficoll on spleen sections, which were also stained for CD169 to delineate the marginal zone. Sections were counterstained with DAPI. Images were acquired on a Zeiss LSM710 confocal microscope equipped with $488 \mathrm{~nm}, 561 \mathrm{~nm}$ and $633 \mathrm{~nm}$ lasers, and with a tunable 2-photon laser. Images were analyzed on Imaris software.

To reveal the clonotypic B cells of $\mathrm{VH} 81 \times \mathrm{Tg}$ mice, we used in house generated antibodies to the heavy chain of VH81x (clone MZ21, rat IgG2a, FITC labeled) and the Vk1C light chain (clone FO27, rat IgG2a, AF647-labeled) ${ }^{14}$.

\section{Immunoblots}


Antibodies to Notch2 (D76A6, Rabbit) and NF-kappaB2 p100/p52 were from Cell Signaling Technologies. Antibodies Taok3 (Clone Ab70297), ADAM10 (EPR5622) were from Abcam. For Western blotting, $500 \mu$ cold RIPA lysis buffer supplemented with protease and phosphatase inhibitors (Roche) were added to freshly collected spleens. These were then homogenized using a rod homogenizer and centrifuged at $14,000 \mathrm{~g} 4^{\circ} \mathrm{C}$ for $15 \mathrm{~min}$. Approximately $10 \mu \mathrm{g}$ of protein was loaded on polyacrylamide gels for Western blot.

\section{PCRs}

mRNA was extracted using the TRIreagent according to manufacturer's specifications (Roche Applied Sciences). cDNA was synthesized with 0,5 mg of mRNA using the High Fidelity cDNA synthesis kit (Roche Applied Sciences). Real time PCR was the conducted on the samples using specific primers and the Roche Syber Green master mix. GAPDH: 5': TGGTGCTTGTCTCACTGACC; 3': TTCAGTATGTTCGGCTTCCC L27: 5': CATGAACTTGCCCATCTCG; 3': TGAAAGGTTAGCGGAAGTGC TAOK3: 5': TTGCATGAAATTGGACATGGGA, 3': CGATGGTGTTAGGATGCTTCAG Deltex1: 5': AGGCGGTGATGAGCAATC, 3': ACCCAGGCAAGAAGTTCACA Hes1: 5':AAAGCCTATCATGGAGAAGAGGCG, 3':GGAATGCCGGGAGCTATCTTTCTT Hes5: 5': AAAGCCTATCATGGAGAAGAGGCG,3' GGAATGCCGGGAGCTATCTTTCTT Hey1:5': ACACTGCAGGAGGGAAAGGTT, 3': CAAACTCCGATAGTCCATAGCCA

\section{Adoptive transfer of T1B and T2B cells}

Transitional $B$ cells were first enriched from the spleens of CD45.2 VH81x Tg mice using CD93-APC staining and magnetic bead enrichment using anti-APC beads and LS columns (Miltenyi). DAPI- living cells were stained for CD23 and surface ADAM10 and sorted using flow cytometry. Subsequently, $1 \times 10^{6} \mathrm{~T} 1 \mathrm{ADAM} 10^{+}$or ADAM10 subsets or T2 cells were mixed with $10 \times 10^{6}$ CD45.1 splenocytes and injected intravenously into CD45.1 Rag2/- recipient mice in $200 \mu$ of phosphate buffered saline. Analysis of splenic $B$ cell subsets was performed 5 days later by perfoming flow cytometry on cell suspensions and donor cells were detected using antibodies to CD45.2 (clone A20 from 
BD Biosciences). We also analyzed the distribution of CD45.2 injected cells on spleen sections also stained for CD169 to delineate the marginal zone.

\section{Generation of mixed bone marrow chimeric mice}

Bone marrow cells were obtained from CD45.1 Taok3 $^{+/+}$and CD45.2 Taok3 ${ }^{-/-}$donor mice. (CD45.1xCD45.2)F1 acceptor mice were irradiated using 10Gy, followed by the intravenous injection of $2 \times 10^{6} \mathrm{Taok}^{+/+}$cells and $2 \times 10^{6} \mathrm{Taok}^{-/-}$cells at least 4 hours after the irradiation. We did not add antibiotics to the drinking water. Mice were euthanized 6-8 weeks after reconstitution. Bone marrow and spleen were analyzed for the presence of $B$ cell progenitors and mature $B$ cells, respectively. The reconstitution was validated using the ratio of CD45.1 wild-type vs CD45.2. Taok3-- cells. 


\section{FIGURE LEGENDS}

Figure 1: Taok3-/- mice lack marginal zone B cells and have reduced humoral responses to T-independent antigens. (a) Taok3 mRNA expression in different tissues of C57BI/6 mice. (b) Percentage of innate immune cells in the spleens of $T a o k 3^{+/+}$and

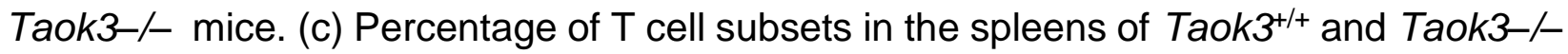
mice. (d) Flow cytometry staining of spleens for CD21/35 hi CD23 lo marginal zone B cells (MZB) and CD21/35- CD23 hi follicular B cells (FoB). (e) Percentage of FoB cells and MZB cells within splenic CD19+ B cells in Taok3 ${ }^{+/+}$and Taok3-/- mice. (f) Percentage of CD93 ${ }^{\text {hi }}$ transitional T1B and T2B cells in the spleens of $\mathrm{Taok}^{+/+}$and Taok3-/- mice. (g) Immunofluorescence staining of $\mathrm{CD} \mathrm{d}^{+}$(green) $\operatorname{lgM}^{+}$(red) marginal zone B cells (double positive MZB cells are yellow) in the spleens of $\mathrm{Taok3}^{+/+}$and Taok3-/ mice. (h) Immunofluorescence uptake of i.v-injected FITC-labeled Ficoll (green) in the spleen of Taok3+/+ and Taok3-/- mice. CD169+ metallophilic macrophages are stained in red. DAPI nuclear counterstaining in blue. (i) Serum IgM titers at baseline and 7 days following TNPFicoll injection in $\mathrm{Taok}^{+/+}$and Taok3-/ mice. (j) Percentage of B1a and B1b cells in peritoneal lavages of $\mathrm{Taok}^{+/+}$and Taok3-/- mice. (k) OD values for phosphorylcholinespecific IgM titers following injection of $\mathrm{Taok}^{+/+}$and Taok3-/- mice with Streptococcus pneumoniae. ${ }^{*} \mathrm{p}<0.05$ (Mann and Whitney test (b, c, e, k)). Data are representative of three experiments (b-f, j; mean + s.e.m.; $n=4-5$ mice per experiment), or one experiment (g-l, k; mean + s.e.m of $n=6$ mice per group in i and k, and at least 4 images in $\mathrm{g}-\mathrm{h}$ ).

Figure 2: The marginal zone B cell defect in Taok3-/- mice is B cell-intrinsic and subject to Taok3 gene dosage. (a) Flow cytometry staining of spleens from chimeric mice to evaluate the ratio between CD45.1 $\mathrm{Taok}^{+/+}$and CD45.2 Taok3-/- cells within the marginal zone B (MZB) cell (upper right plot) and the follicular B (FoB) cell (lower right plot) gates. The numbers adjacent to the gates represent the percentage of cells within the gates. (b) Quantification of the ratios between CD45.1 Taok3 ${ }^{+/+}$and CD45.2 Taok3/ cells on the populations gated as in (a). (c) Gene-trap reversal recovery of marginal zone B cells in Taok3-/- mice crossed to mb1Cre mice was analyzed by flow cytometry. (d) Quantification of the Gene-trap reversal recovery shown in (d) in different mouse 
genotypes. Data are representative of three experiments (b), two experiments (d-e), or a pool of 2 independent experiments (c); $n=5-7$ mice per experiment).

\section{Figure 3: Taok3-/- transitional B cells have a defect in Notch activation.}

(a) Quantification of Notch target gene induction in CD93 ${ }^{\text {hi }}$ transitional B cells from Taok3 ${ }^{+/+}$and Taok3-/ mice after 4 hours of culture on OP9-Dll1 in the absence of BAFF. (b) Flow cytometry analysis of the induction of CD21/35 expression on Transitional B cells cultured on OP9-GFP or OP9-DII1 for 5 days in the presence of BAFF. (c) Flow cytometry analysis (upper panel) and quantification (lower panel) of marginal zone B cells in the spleens of $\mathrm{Taok}^{+/+}$and Taok3-/- mice crossed to Irf4 ${ }^{-/-}$mice. (d) Immunofluorescence staining of marginal zone B cells in the spleen of Taok $3^{+/+}$and Taok3-/- mice crossed to Irf4-/- mice. Data are representative of two experiments (b-d). d: mean + s.e.m.; $n=2-6$ mice per experiment. i, at least 3 images per group were acquired.

Figure 4: Taok3 controls the surface expression of ADAM10. (a) Immunofluorescence staining of CD23 (red) in the spleen of $\mathrm{Taok}^{+/+}$and Taok3-/- mice. White, CD169+ metallophilic macrophages. Blue, DAPI nuclear counterstaining. Scale bar, 100 $\mu \mathrm{m}$. (b) Flow cytometry analysis of CD23 expression on Taok3 ${ }^{+/+}$and Taok3-/- splenic Follicular

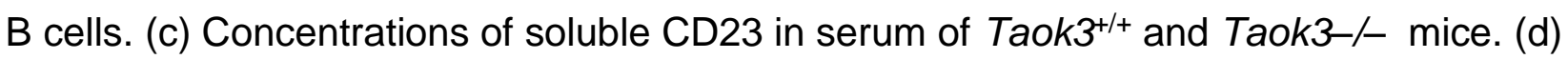
Concentrations of soluble CD23 in serum of $\mathrm{Taok3}^{+/+}$and Taok3-/ - mice mice treated or not with 19G5 antibody. (e) Flow cytometry analysis of surface ADAM10 expression on non-permeabilized splenic CD93 ${ }^{\text {hi }}$ transitional B cells of Taok3 ${ }^{+/+}$and Taok3-/ mice. (f) Flow cytometry analysis (left panels) and quantification (right panel) of ADAM10 expression on splenic CD93 ${ }^{\text {hi }}$ transitional B cells of Taok $^{+/+}$and Taok3-/- mice crossed to Irf4 ${ }^{-1-}$ mice. $(\mathrm{g})$ Correlation between the percentage of marginal zone $\mathrm{B}$ cells and the percentage of ADAM10+ $B$ cells in the spleen. ${ }^{*} p<0.05$ (Mann and Whitney test $(c, d, f)$ ). Data are representative of at least three experiments $(b, e)$, two experiments $(c, d)$, or one experiment (f). (c, d): mean + s.e.m.; $n=4-6$ mice per experiment. (f): mean + s.e.m.; $n=$ 2 mice per experiment. 
Figure 5: ADAM10 expression on transitional $B$ cells marks commitment to the MZB cell fate. (a) Immunofluorescence staining of ADAM10 (red) on purified CD93+

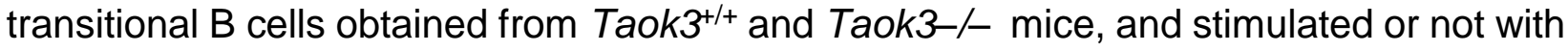
anti-lgM $F\left(a b^{\prime}\right)_{2}$ fragments for 15 minutes. Blue, nuclear counterstaining. Scale bar, $5 \mu \mathrm{m}$. (b) Immunofluorescence staining of marginal zone B cells (green) in the spleens of

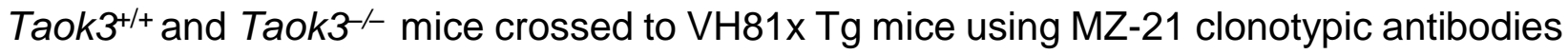
recognizing the heavy chain Ig transgene. Blue, nuclear counterstaining. Scale bar, 100

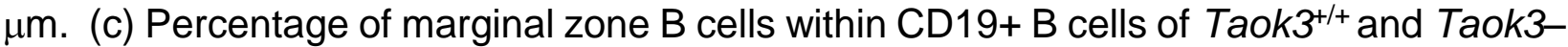
/ mice crossed to VH81x Tg mice. (d) Flow cytometry analysis of the phenotype of clonotype+ cells in the spleen of Taok3 ${ }^{+/+}$and Taok3-/- mice crossed to VH81x Tg mice. (e) Percentage of clonotype ${ }^{+}$cells within B cells in the spleen of $\mathrm{Taok3}^{+/+}$and Taok3-/mice crossed to VH81x Tg mice. (f) Flow cytometry analysis of ADAM10 expression on splenic $\mathrm{CD} 3^{\text {hi }}$ transitional B cells of $T a o k 3^{+/+}$and Taok3-/- mice crossed to VH81x Tg mice. (g) Flow cytometry staining of the spleens of Rag ${ }^{/-}$recipients treated as in (f) for the presence of $C D 21 / 35^{\text {hi }} C D 23^{\text {lo }}$ marginal zone $B$ cells and CD21/35- CD23 $3^{\text {hi }}$ Follicular $B$ cells. (h) Immunofluorescent staining of ADAM10+ CD45.2 (red) transferred T1B cells in the spleen of $\mathrm{Rag}^{/-}$recipient mice. Green, CD169+ metallophilic macrophages. Blue, DAPI nuclear counterstaining. Scale bar, $100 \mu \mathrm{m}$. ${ }^{*} \mathrm{p}<0.05$ (Mann and Whitney test (c, e). Data are representative of at least two experiments. (c, e): mean + s.e.m.; $n=4-12$ mice per experiment. 


\section{REFERENCES}

Updated style is available in the ThomsonReuters website. You can download the update style from here: http://endnote.com/downloads/style/nature-immunology If you have any other questions about their product, please reach out to them at http://ipscience.thomsonreuters.com/support/

1. Martin F, Oliver AM, Kearney JF. Marginal zone and B1 B cells unite in the early response against T-independent blood-borne particulate antigens. Immunity 2001, 14(5): 617-629.

2. Tanigaki K, Han H, Yamamoto N, Tashiro K, Ikegawa M, Kuroda K, et al. Notch-RBP-J signaling is involved in cell fate determination of marginal zone B cells. Nat Immunol 2002, 3(5): 443-450.

3. Kuroda K, Han H, Tani S, Tanigaki K, Tun T, Furukawa T, et al. Regulation of marginal zone B cell development by MINT, a suppressor of Notch/RBP-J signaling pathway. Immunity 2003, 18(2): 301-312.

4. Tan JB, Xu K, Cretegny K, Visan I, Yuan JS, Egan SE, et al. Lunatic and manic fringe cooperatively enhance marginal zone B cell precursor competition for delta-like 1 in splenic endothelial niches. Immunity 2009, 30(2): 254-263.

5. Fasnacht N, Huang HY, Koch U, Favre S, Auderset F, Chai Q et al. Specific fibroblastic niches in secondary lymphoid organs orchestrate distinct Notch-regulated immune responses. J Exp Med 2014, 211(11): 2265-2279.

6. Saito T, Chiba S, Ichikawa M, Kunisato A, Asai T, Shimizu K, et al. Notch2 is preferentially expressed in mature B cells and indispensable for marginal zone B lineage development. Immunity 2003, 18(5): 675-685.

7. Witt CM, Won WJ, Hurez V, Klug CA. Notch2 haploinsufficiency results in diminished B1 B cells and a severe reduction in marginal zone B cells. J Immunol 2003, 171(6): 2783-2788.

8. Moran ST, Cariappa A, Liu H, Muir B, Sgroi D, Boboila C, et al. Synergism between NFkappa B1/p50 and Notch2 during the development of marginal zone B lymphocytes. J Immunol 2007, 179(1): 195-200.

9. Wu L, Maillard I, Nakamura M, Pear WS, Griffin JD. The transcriptional coactivator Maml1 is required for Notch2-mediated marginal zone B-cell development. Blood 2007, 110(10): 3618-3623. 
10. Gibb DR, El Shikh M, Kang DJ, Rowe WJ, El Sayed R, Cichy J, et al. ADAM10 is essential for Notch2-dependent marginal zone B cell development and CD23 cleavage in vivo. J Exp Med 2010, 207(3): 623-635.

11. Hampel F, Ehrenberg S, Hojer C, Draeseke A, Marschall-Schroter G, Kuhn R, et al. CD19-independent instruction of murine marginal zone B-cell development by constitutive Notch2 signaling. Blood 2011, 118(24): 6321-6331.

12. Simonetti G, Carette A, Silva K, Wang H, De Silva NS, Heise N, et al. IRF4 controls the positioning of mature $\mathrm{B}$ cells in the lymphoid microenvironments by regulating NOTCH2 expression and activity. J Exp Med 2013, 210(13): 2887-2902.

13. Hozumi K, Negishi N, Suzuki D, Abe N, Sotomaru Y, Tamaoki N, et al. Delta-like 1 is necessary for the generation of marginal zone B cells but not T cells in vivo. Nat Immunol 2004, 5(6): 638-644.

14. Martin F, Kearney JF. Positive selection from newly formed to marginal zone B cells depends on the rate of clonal production, CD19, and btk. Immunity 2000, 12(1): 3949.

15. Wen L, Brill-Dashoff J, Shinton SA, Asano M, Hardy RR, Hayakawa K. Evidence of marginal-zone B cell-positive selection in spleen. Immunity 2005, 23(3): 297-308.

16. Carey JB, Moffatt-Blue CS, Watson LC, Gavin AL, Feeney AJ. Repertoire-based selection into the marginal zone compartment during B cell development. J Exp Med 2008, 205(9): 2043-2052.

17. Cariappa A, Tang M, Parng C, Nebelitskiy E, Carroll M, Georgopoulos K, et al. The follicular versus marginal zone B lymphocyte cell fate decision is regulated by Aiolos, Btk, and CD21. Immunity 2001, 14(5): 603-615.

18. Pillai S, Cariappa A. The follicular versus marginal zone B lymphocyte cell fate decision. Nat Rev Immunol 2009, 9(11): 767-777.

19. Boyce KJ, Andrianopoulos A. Ste20-related kinases: effectors of signaling and morphogenesis in fungi. Trends Microbiol 2011, 19(8): 400-410.

20. Chuang HC, Wang X, Tan TH. MAP4K Family Kinases in Immunity and Inflammation. Adv Immunol 2016, 129: 277-314.

21. Guinamard R, Okigaki M, Schlessinger J, Ravetch JV. Absence of marginal zone B cells in Pyk-2-deficient mice defines their role in the humoral response. Nat Immunol 2000, 1(1): 31-36. 
22. Balazs M, Martin F, Zhou T, Kearney J. Blood dendritic cells interact with splenic marginal zone B cells to initiate T-independent immune responses. Immunity 2002, 17(3): 341-352.

23. Kin NW, Crawford DM, Liu J, Behrens TW, Kearney JF. DNA microarray gene expression profile of marginal zone versus follicular B cells and idiotype positive marginal zone B cells before and after immunization with Streptococcus pneumoniae. J Immunol 2008, 180(10): 6663-6674.

24. Arnon TI, Horton RM, Grigorova IL, Cyster JG. Visualization of splenic marginal zone B-cell shuttling and follicular B-cell egress. Nature 2013, 493(7434): 684-688.

25. Cinamon G, Matloubian M, Lesneski MJ, Xu Y, Low C, Lu T, et al. Sphingosine 1phosphate receptor 1 promotes B cell localization in the splenic marginal zone. Nat Immunol 2004, 5(7): 713-720.

26. Lu TT, Cyster JG. Integrin-mediated long-term B cell retention in the splenic marginal zone. Science 2002, 297(5580): 409-412.

27. Hobeika E, Thiemann S, Storch B, Jumaa H, Nielsen PJ, Pelanda R, et al. Testing gene function early in the B cell lineage in mb1-cre mice. Proc Natl Acad Sci U S A 2006, 103(37): 13789-13794.

28. Descatoire M, Weller S, Irtan S, Sarnacki S, Feuillard J, Storck S, et al. Identification of a human splenic marginal zone B cell precursor with NOTCH2-dependent differentiation properties. J Exp Med 2014, 211(5): 987-1000.

29. Roundy KM, Jacobson AC, Weis JJ, Weis JH. The in vitro derivation of phenotypically mature and diverse B cells from immature spleen and bone marrow precursors. Eur J Immunol 2010, 40(4): 1139-1149.

30. Weskamp G, Ford JW, Sturgill J, Martin S, Docherty AJ, Swendeman S, et al. ADAM10 is a principal 'sheddase' of the low-affinity immunoglobulin E receptor CD23. Nat Immunol 2006, 7(12): 1293-1298.

31. De Strooper B, Vassar R, Golde T. The secretases: enzymes with therapeutic potential in Alzheimer disease. Nat Rev Neurol 2010, 6(2): 99-107.

32. Gupta N, Wollscheid B, Watts JD, Scheer B, Aebersold R, DeFranco AL. Quantitative proteomic analysis of $B$ cell lipid rafts reveals that ezrin regulates antigen receptormediated lipid raft dynamics. Nat Immunol 2006, 7(6): 625-633.

33. Karlsson MC, Guinamard R, Bolland S, Sankala M, Steinman RM, Ravetch JV. Macrophages control the retention and trafficking of B lymphocytes in the splenic marginal zone. J Exp Med 2003, 198(2): 333-340. 
34. Moriyama Y, Sekine C, Koyanagi A, Koyama N, Ogata H, Chiba S, et al. Delta-like 1 is essential for the maintenance of marginal zone B cells in normal mice but not in autoimmune mice. Int Immunol 2008, 20(6): 763-773.

35. Chen X, Martin F, Forbush KA, Perlmutter RM, Kearney JF. Evidence for selection of a population of multi-reactive B cells into the splenic marginal zone. Int Immunol 1997, 9(1): 27-41.

36. Pillai S, Cariappa A, Moran ST. Marginal zone B cells. Annu Rev Immunol 2005, 23: 161-196.

37. Casola S, Otipoby KL, Alimzhanov M, Humme S, Uyttersprot N, Kutok JL, et al. B cell receptor signal strength determines B cell fate. Nat Immunol 2004, 5(3): 317-327.

38. Deng W, Cho S, Su PC, Berger BW, Li R. Membrane-enabled dimerization of the intrinsically disordered cytoplasmic domain of ADAM10. Proc Natl Acad Sci U S A 2014, 111(45): 15987-15992.

39. Xu P, Liu J, Sakaki-Yumoto M, Derynck R. TACE activation by MAPK-mediated regulation of cell surface dimerization and TIMP3 association. Sci Signal 2012, 5(222): ra34.

40. Kapfhamer D, King I, Zou ME, Lim JP, Heberlein U, Wolf FW. JNK pathway activation is controlled by Tao/TAOK3 to modulate ethanol sensitivity. PLoS One 2012, 7(12): e50594.

41. Srivastava B, Quinn WJ, 3rd, Hazard K, Erikson J, Allman D. Characterization of marginal zone B cell precursors. J Exp Med 2005, 202(9): 1225-1234.

42. Dammers PM, de Boer NK, Deenen GJ, Nieuwenhuis P, Kroese FG. The origin of marginal zone B cells in the rat. Eur J Immunol 1999, 29(5): 1522-1531.

43. Holmes R, Zuniga-Pflucker JC. The OP9-DL1 system: generation of T-lymphocytes from embryonic or hematopoietic stem cells in vitro. Cold Spring Harb Protoc 2009, 2009(2): pdb prot5156. 